\title{
Polychlorinated Biphenyls in the Centralized Wastewater Treatment Plant in a Chemical Industry Zone: Source, Distribution, and Removal
}

\author{
Min Yao, ${ }^{1,2}$ Zhongjian Li, $^{1}$ Xingwang Zhang, ${ }^{1}$ and Lecheng Lei ${ }^{1}$ \\ ${ }^{1}$ Key Laboratory of Biomass Chemical Engineering of Ministry of Education, Zhejiang University, Hangzhou 310027, China \\ ${ }^{2}$ Faculty of Architectural, Civil Engineering and Environment College, Ningbo University, Ningbo 315211, China \\ Correspondence should be addressed to Lecheng Lei; lclei@zju.edu.cn
}

Received 13 March 2014; Revised 8 June 2014; Accepted 10 July 2014; Published 5 August 2014

Academic Editor: Davide Vione

Copyright (c) 2014 Min Yao et al. This is an open access article distributed under the Creative Commons Attribution License, which permits unrestricted use, distribution, and reproduction in any medium, provided the original work is properly cited.

\begin{abstract}
Polychlorinated biphenyls (PCBs) could be dissolved in wastewater or adsorbed on particulate. The fate of PCBs in wastewater is essential to evaluate the feasibility of wastewater treatment processes and the environmental risk. Here dissolved and adsorbed concentrations of twenty concerned PCB congeners and total PCBs have been measured in the centralized wastewater treatment plant of a chemical industry zone in Zhejiang, China. It was found that the dyeing chemical processes were the main source of PCBs, which contributed more than 13.6\%. The most abundant PCB was PCB-11 in the liquid and solid phase of each treatment stage, accounting for more than $60 \%$ of the total 209 PCBs. Partitioning behavior of PCBs between the dissolved and adsorbed phases suggested that $\mathrm{Di}$-CBs were the dominant isomers $(>70 \%)$ and more than $89.8 \%$ of them was adsorbed on the particles and sludge. The total removal efficiency of $\sum 209$ PCBs was only $23.2 \%$ throughout the whole treatment process. A weak correlation was obtained between the individual PCB concentration and their $\log K_{\mathrm{ow}}$ in primary sedimentation, anaerobic hydrolysis, aerobic bioprocess stage, and the whole treatment process.
\end{abstract}

\section{Introduction}

Polychlorinated biphenyls (PCBs) are a class of significant persistent organic pollutants (POPs) that consist of 209 congeners, being of increasing global concern because of their high toxicity, resistance to biodegradation, biological accumulation and long-range transport [1]. Although PCBs production in most countries has been banned since the 1970s and 1980s, PCBs contaminations remain ubiquitous and coexist in most environmental matrices, including water, sediment, soil, and air all over the world [2-4]. Exposure to PCBs can cause neurological disorder, reproductive toxicity, endocrine disruption, cancer, and deformity, even at extremely low concentrations [5]. Thus, it is important and necessary to study the source, distribution, and removal of PCBs in the environment.

There are various sources of existing PCBs in the environment, most of which were accumulated during the period 1950 to 1983 , due to their worldwide use as lubricants, heat transfer agents, paint additives, and insulating media in capacitors, and voltage regulations. Also, PCBs can be generated and introduced into the environment as byproducts from waste incineration and various chemical industrial processes [6]. Therefore, tracing the source of PCBs is very important to study their transport and fate in the environment [7]. Recently, it is reported that the wastewater treatment plants (WWTPs) are another potential source of PCBs to the ambient environment $[6,8-11]$. Therefore, increasing attention has been paid to the fate of PCBs in WWTPs. Katsoyiannis and Samara [12] found that more than $50 \%$ of the total seven indicator PCB congeners (PCB-28, 52, 101, 118, 138,153 , and 180) were absorbed in sludge and the concentration of the PCBs in wastewater was decreased from 1000 to $250 \mathrm{ng} / \mathrm{L}$ after treatment (samples were from a WWTP in Thessaloniki, northern Greece). However, Bergqvist et al. [10] found that the concentrations of $\sum$ PCBs (seven indicator PCBs and PCB-47, 105, 156) increased from 0.3 to $1 \mathrm{ng} / \mathrm{L}$ in Umeå (Sweden) and from 9 to $34 \mathrm{ng} / \mathrm{L}$ in Šiauliai 


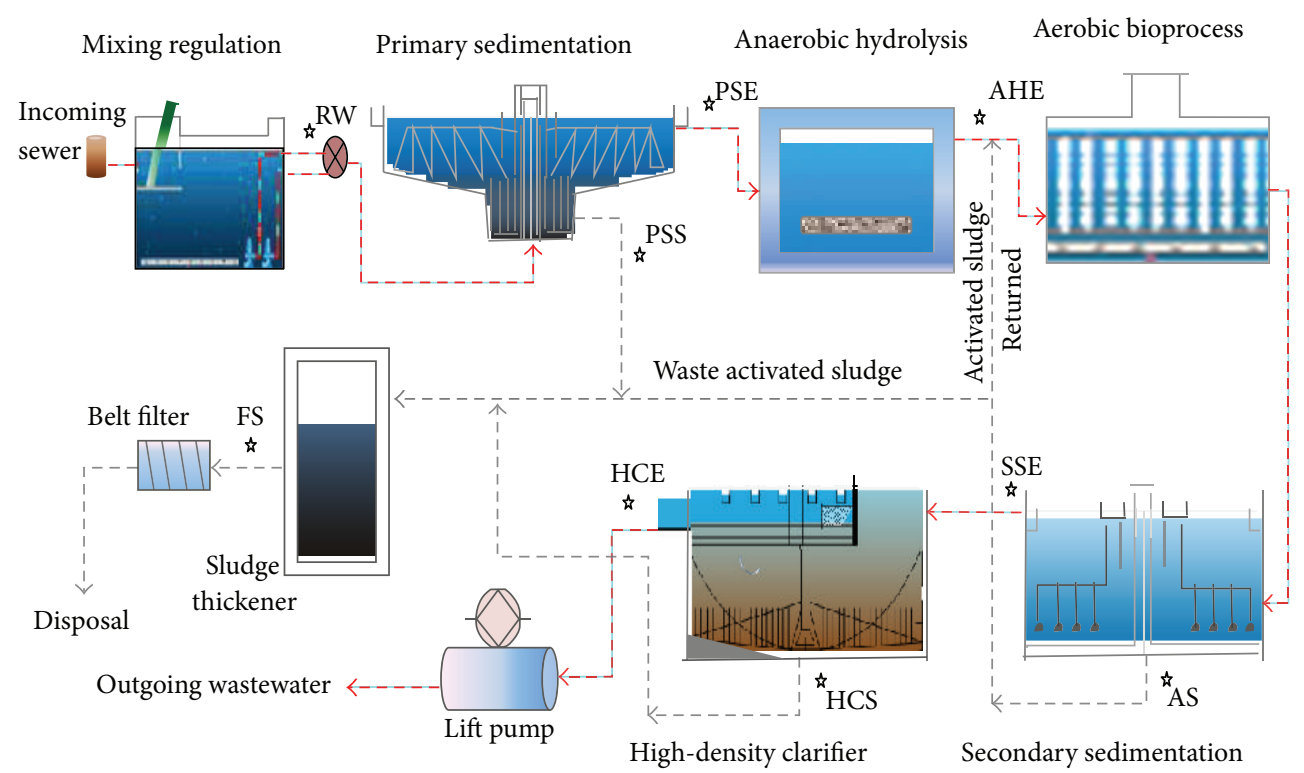

FIGURE 1: Flow chart of the centralized WWTP in Zhejiang, China (ఓ: location of samplings). RW, raw wastewater; PSE, primary sedimentation effluent; AHE, anaerobic hydrolysis effluent; SSE, secondary sedimentation effluent; HCE, high-density clarifier effluent; PSS, primary sedimentation sludge; AS, activated sludge; HCS, high-density clarifier sludge; and FS, final sludge.

(Lithuania) WWTP during the treatment process. Based on the above-mentioned two papers, it is very likely that different wastewater components and wastewater treatment processes have significant effect on the PCBs fate. In China, owing to the rapid development in manufacturing industries, the pollutants in wastewater are increasing dramatically, which increased the variety and complexity of PCBs in wastewater in WWTPs. However, few detail data are available for the fate and removal of PCBs in the WWTPs in China taking both PCBs distribution in water and absorption in sludge into account.

The aim of our study was to thoroughly investigate the fate of PCBs in the conventional wastewater treatment processes. Here, a WWTP treating dyeing chemicals and domestic wastewater in Zhejiang province, China, was chosen in this project. Firstly, we investigated the PCBs in the WWTP contributed by the dyeing chemical industry. Then the indicator PCBs, dioxin-like PCB congeners and three lightly chlorinated biphenyls (PCB-11, 15, 19) and total PCBs (from Mono-CBs to Deca-CBs) were quantitatively analyzed both in wastewater and sludge at each treatment stage in the WWTP. The distribution of PCBs between the dissolved and adsorbed phases in the influent and effluent of each treatment stage was investigated as well. Furthermore, PCBs removal efficiencies at each treatment stage and the key removal mechanism were also studied. Our work will provide a deeper understanding of the PCBs fate in WWTP and a theoretical basis for the source control.

\section{Materials and Methods}

\subsection{Plant Description and Sampling}

2.1.1. Plant Description. The centralized wastewater treatment plant (WWTP) is located in a chemical industry zone in Zhejiang province, China. The WWTP is located outdoors and processes $90,000-120,000 \mathrm{~m}^{3}$ of raw wastewater per day. About $70 \%$ of the total flow is contributed by industrial activities, especially by dyeing chemical industries in the zone, as well as a small amount of domestic sewage. The influents of wastewater through pipelines first pass screening and grit and grease chambers, and then are fully mixed in the mixing regulation tank to be the raw wastewater (i.e., mixed influent). The wastewater treatment processes include primary sedimentation using chemical flocculants (polyaluminum chloride, $\mathrm{PAC}$ ), anaerobic/aerobic (A/O) biochemical treatment (including anaerobic hydrolysis process by anaerobic biofilter and aerobic bioprocess by activated sludge), secondary sedimentation for the settlement of activated sludge, and high-density clarifier by ferrate oxidation. The flow chart of the plant is shown in Figure 1.

2.1.2. Sampling Sites. The wastewater, suspended particulate matter (SPM), and sludge samples were taken from the outlet of each processing stage during late October and early November 2010 (October 26 and November 6). Duplicate samples of wastewater were collected along the treatment system to study the sampling reproducibility, namely, the mixed influent of raw wastewater in the mixing regulation tank (RW), the effluents of primary sedimentation, anaerobic hydrolysis, aerobic bioprocess, secondary sedimentation, and high-density clarifier tanks (PSE, AHE, SSE, and HCE, resp.). Grab samples of sludge were collected from the primary sedimentation tank (PS), the recirculation stream (activated sludge, AS), high-density clarifier tank (HCS), and sludge thickener tank (final sludge, FS) concurrently with the wastewater samples. All sampling containers were in sequence washed with water, acetone, dichloromethane, and the wastewater samples before use. 
Furthermore, in order to estimate the industrial sources of PCBs, two wastewater samples were collected from the largest dyeing chemical group in this zone, which mainly produced disperse, reactive, acid, vat dyestuff, or pigments and intermediates. One sample was the mother liquor produced during the pigment synthesis process and the other was the treated effluent of the mother liquor entering the WWTP. The amounts of wastewater discharged into the WWTP were about $8,000 \mathrm{~m}^{3}$ per day.

2.2. Chemicals and Reagents. All solvents, including acetone, n-hexane, methanol, and dichloromethane, were of HPLC grade and purchased from Tedia Co., USA. Water was purified with an ultrapure water system (Purelab UHQ, Elga LabWater, UK). Silica gel (100-200 mesh, reagent grade, Qingdao Ocean Chemical Reagent Co., China), anhydrous sodium sulfate (analytical grade, Chongqing Kelong Chemical Reagent Co., China), and glass fiber filters $(0.45 \mu \mathrm{m}$ effective pore sizes, Shanghai Mosu Scientific Equipment Co., China) were baked in a furnace oven at $450^{\circ} \mathrm{C}$ for $4 \mathrm{~h}$ prior to use.

PCB calibration standard solutions, ${ }^{13} \mathrm{C}$-labeled surrogate standards ( ${ }^{13} \mathrm{C}-\mathrm{PCB}-14,65$ and 166), and injection standards $\left({ }^{13} \mathrm{C}-\mathrm{PCB}-9,52,101,138\right.$ and 194) complying with US EPA method 1668A for PCBs analysis were purchased from Cambridge Isotope Laboratories in USA. Here, the mainly concerned PCBs were 20 individual PCB congeners and total PCBs (from Mono-CBs to Deca-CBs). The individual congeners included six indicator congeners (PCB-28, 52, 101, 118, 138,153 , and 180), ten dioxin-like PCB (PCB-77, 81, 105, 114, $123,126,156,167,169$, and 189) and three lightly chlorinated biphenyls (PCB-11, 15, and 19), respectively. The working standard solutions were prepared by diluting appropriate volumes of the standard PCB mixture with HPLC-grade nhexane.

2.3. Sample Extraction and Purification. $1 \mathrm{~L}$ of wastewater samples was filtered using glass fiber filter and spiked with $200 \mathrm{ng}$ of ${ }^{13} \mathrm{C}$-labeled surrogate standards and then extracted three times under ultrasonic conditions using liquid-liquid extraction method. The total extracted volume was $180 \mathrm{~mL}$ of dichloromethane/hexane $(1: 1, \mathrm{v} / \mathrm{v})$ and the extracts were concentrated to $2 \mathrm{~mL}$ and subjected to a solvent exchange to hexane by a rotary evaporator (RE-52AA, Yarong, Shanghai, China). The concentrated extracts were sequentially subject to multilayer silica gel, basic alumina, and florisil chromatography columns for further cleanup, following the published procedures $[9,13-15]$. The multilayered silica gel column was self-made column, which was packed from bottom to top with $1 \mathrm{~g}$ of activated silica, $4 \mathrm{~g}$ of basic silica $(1.2 \% \mathrm{w} / \mathrm{w})$, $1 \mathrm{~g}$ of activated silica, $8 \mathrm{~g}$ of acid silica $(30 \% \mathrm{w} / \mathrm{w}), 2 \mathrm{~g}$ of activated silica, and $4 \mathrm{~g}$ of anhydrous sodium sulfate. The columns were preserved in dichloromethane/hexane $(1: 1$, $\mathrm{v} / \mathrm{v}$ ) before loading the extracts. Elution of the samples was carried out at a rate of $1 \mathrm{~mL} / \mathrm{min}$ under vacuum with $5 \%$ dichloromethane/hexane (v/v). Then the eluent was collected and evaporated to near dryness with rotary evaporator. Hexane solvent was introduced as a replacement and then concentrated to $20 \mu \mathrm{L}$ with gentle stream of nitrogen. Then, ${ }^{13} \mathrm{C}$-labeled internal standards were added prior to the GC injection for quantitative analysis. All samples were analyzed in duplicate and the average concentrations (means $\pm \mathrm{SD}$, $n=2)$ were reported.

The SPM in the wastewater was separated by glass fiber filters and the sludge was dehydrated by centrifugation. The filters and sludge were freeze dried until constant weights were maintained. Solid samples were accurately weighted and spiked with $1 \mathrm{ng}$ of ${ }^{13} \mathrm{C}$-labeled surrogate standards and then Soxhlet-extracted for $24 \mathrm{~h}$ with $400 \mathrm{~mL}$ of dichloromethane/acetone $(1: 1, \mathrm{v} / \mathrm{v})$. The extracts were concentrated to about $5 \mathrm{~mL}$ and subjected to a solvent exchange to hexane. The concentrated extracts were subsequently purified and fractionated following the above mentioned method under identical conditions [16].

2.4. GC/MS Analysis and Quality Control. PCBs were analyzed by isotope dilution method and the analysis procedure was similar to the method described by Yang et al. [15]. The quantification of PCBs extracts in the wastewater, SPM, and sludge samples was performed on an Agilent 6890A gas chromatograph (GC) coupled with 5795C inert mass spectrometer (MS) detector (Agilent Technologies, USA) with an electron impact (EI) ion source. The MS was operated in selective ion monitoring (SIM) mode. Helium was used as carrier gas with flow rate of $1.2 \mathrm{~mL} / \mathrm{min}$. Exactly $1 \mu \mathrm{L}$ of extract solution was injected with 16-sample autoinjector in splitless mode into a DB-5MS capillary column $(60 \mathrm{~m} \times 0.25 \mathrm{~mm}$ i.d. $\times 0.25 \mu \mathrm{m}$ film thickness, J\&W Scientific, USA). The injector temperature and source temperature were 280 and $250^{\circ} \mathrm{C}$, respectively. The oven temperature program was as follows: $110^{\circ} \mathrm{C}$ held for $3 \mathrm{~min}, 110-150^{\circ} \mathrm{C}$ at $10^{\circ} \mathrm{C} / \mathrm{min}, 150-270^{\circ} \mathrm{C}$ at $2.5^{\circ} \mathrm{C} / \mathrm{min}$ held for $5 \mathrm{~min}$, and $270-320^{\circ} \mathrm{C}$ at $2.5^{\circ} \mathrm{C} / \mathrm{min}$ held for $10 \mathrm{~min}$.

The blank, blank spike, and parallel samples were introduced for quality assurance and quality control. The background concentrations were negligible, due to their significantly lower levels compared to actual samples ( $<3 \%)$. Recoveries of added ${ }^{13} \mathrm{C}$-labeled PCB-14, PCB-65, and PCB-166 surrogate standards to wastewater samples prior to extraction averaged $84 \pm 6 \%, 88 \pm 6 \%$, and $94 \pm 4 \%$, respectively. Recoveries of added ${ }^{13}$ C-PCB-14, PCB-65 and PCB-166 to SPM and sludge samples prior to extraction averaged $68 \pm$ $15 \%, 72 \pm 15 \%$, and $88 \pm 10 \%$, respectively. The recoveries meet the requirements of US EPA method 1668A [14].

\section{Results and Discussion}

\subsection{Source Analysis of PCBs from Dyeing Chemical Industry} Entering the WWTP. PCBs entering the centralized WWTP can be mainly associated with direct discharges from industrial emissions in the investigated zone. In order to identify the main industrial emissions as PCB source to the WWTP, we investigated the profiles of the factories or companies in the investigated zone. This zone is a professional development zone featuring as fine chemical industry, focusing on producing of pharmaceuticals and the intermediates, pigments 
and dyes, textile dyeing and finishing, biochemical, inorganic chemicals, and other specialty chemicals. The wastewater produced during the chemical processes was discharged into the WWTP after simple treatment. In this zone, the wastewater discharged by pigment and dyeing chemical industries accounts for about $70 \%$ of the total industrial wastewater. Data provided by US EPA and other workers indicate that PCBs have been found in various color dyestuff and pigments [3, 17-19]. Rodenburg and colleagues knew PCB-11 was linked to the manufacture of diarylide yellow pigments [3]. Hu and Hornbuckle detected more than 50 PCB congeners in azo and phthalocyanine pigments [18]. Litten et al. also found high levels of PCBs in wastewater in New York harbor and traced them to effluent from a pigment manufacturing plant [17]. Thus, we mainly detected the PCBs in dyeing chemical wastewater discharged by the largest chemical industrial group and identified the contribution of PCBs to the WWTP by the group.

The PCB species and concentrations in wastewater depend on the raw materials and the process of dyestuff or pigments production. It is investigated that the dyeing chemical industrial group mainly produces disperse, reactive, vat pigments, and some intermediates, which are mainly azo pigments with polycyclic ketone structures and produced through a reaction sequence of diazotization (coupling to afford the azo group, $-\mathrm{N}=\mathrm{N}-$ ), condensation, close-loop, chlorination, oxidation and reduction, and so forth [20]. During the synthesis of these productions, there are various derivatives of polycyclic hydrocarbons used as the precursors, such as alkylbenzenes, naphthol, anthrone, anthraquinone, acenaphthene, and quinone [21]. Therefore, with high temperature and humidity in the dye synthesis processes, it is easy to generate PCBs and other POPs byproducts. Based on the concentrations and water quantity, the average contribution of each PCB congener was calculated and listed in Table 1. It is shown that lightly chlorinated biphenyls were the main PCB species, and PCB-11, 77, 28, and 15 were the most abundant congeners produced during the dye manufacturing processes. The contribution of $\sum 209$ PCBs produced by this dyeing chemical industrial group to the WWTP was $13.6 \%$. The three greatest contributors were Di-CBs, Hexa-CBs, and Nona-CBs with the contribution percent of $15.4 \%, 15.2 \%$, and $11.6 \%$, respectively.

\subsection{PCB Concentrations in Wastewater Samples of the WWTP.}

The results of PCB concentrations in wastewater samples in the influent and effluent of each WWTP treatment stage are presented in Table 2. As shown, the concerned 20 PCB congeners and $\mathrm{PCB}$ isomers were detectable in the RW samples. The most abundant PCB entering the WWTP in RW was PCB-11 with mean concentration of $10321.6 \mathrm{pg} / \mathrm{L}$, accounting for $66.1 \%$ of the total 209 PCBs. The less abundant PCBs were still the lightly chlorinated biphenyls, including PCB-15, two indicator PCB congeners (PCB-52 and PCB28 ), and one dioxin-like PCB congener (PCB-77) with mean concentrations of 241.6, 249.1, 173.5, and $114.4 \mathrm{pg} / \mathrm{L}$, respectively. The concentrations of total 209 PCBs were two or three orders of magnitude lower than the ones observed in the previous studies [12, 22, 23], which indicated the risk caused by dissolved PCBs was relatively low around the WWTP. Compared with the data in the literature of Vogelsang et al. [24], the concentration of indicator PCBs was three times as ours.

In PSE, AHE, SSE, and HCE samples, the presence of most PCB congeners was in general similar to that in RW samples, with PCB-11 being the most abundant compound and PCB-15, PCB-28, PCB-52, and PCB-77 being the less abundant compounds. PCB-126, PCB-169, and PCB-189 were not detected in the effluent samples of each treatment stage, although found in RW. PCB-81 and PCB-180 were not detected in HCE samples. Interestingly, $\sum 209$ PCBs mean concentration in AHE samples was higher than that in PSE samples. In detail, the concentration of PCB-28, PCB-52, and PCB-77 in AHE samples suddenly increased by $270 \%, 35.7 \%$, and $52.3 \%$, respectively. In anaerobic hydrolysis stage, the anaerobic microbes or anaerobic sludge always stay in the anaerobic fluidized bed without replacement. Thus, the PCBs results may be associated with the role of anaerobic microbes and the release of PCBs from solid to liquid phase.

\subsection{PCB Concentrations in SPM and Sludge Samples of the} WWTP. Table 3 summarizes the mean concentrations (ng/g dry weight) of the 20 PCB congeners and PCB isomers in the SPM and sludge samples. Here five PCB congeners were not detected in the SPM of raw wastewater, that is, PCB-15, PCB-19, and three dioxin-like PCBs (PCB-126, PCB-156, and PCB-169). All the 20 PCBs were detectable in the solid-phase samples taken from following stage. As seen in Tables 2 and 3 , the levels of PCB congeners in the solid phase were closely related to the liquid concentrations in the influent. The higher concentrations of PCBs in the influent, the higher levels in SPM and sludge samples from effluent. For example, PCB-11 was the most abundant PCBs both in liquid and in solid phase with a high proportion of more than $60 \%$.

In primary sedimentation stage, the concentrations of total PCBs were nearly 2 times that in SPM of raw wastewater, due to adsorption and sedimentation by PAC flocculants. High levels of PCBs were detected in anaerobic SPM and aerobic activated sludge, indicating that the microbial sludge had a strong biosorption and bioaccumulation for PCBs, which made the contribution of biotransformation, biodegradation, and volatilization obscure. Therefore, sorption of PCBs onto sludge was the most likely removal mechanism in the primary and biochemical treatment stages. In highdensity clarifier stage, PCBs concentrations in effluent and sludge samples decreased more significantly than the ones in the aerobic bioprocess stage owing to the oxidation (although no evidence is available in the literature of ferrate reaction with PCBs) or the coagulation by ferrate, because ferrate at $\mathrm{pH}<7$ generates $\mathrm{Fe}$ (III) ions with excellent flocculation capability and $\mathrm{Fe}(\mathrm{OH})_{3}$ that is a good adsorbent [25].

The mean concentrations of PCBs in the final sludge of the centralized WWTP were also within the range of reported values in Table 3. $\sum 209$ PCBs mean concentration $(6432.3 \mathrm{ng} / \mathrm{g} \mathrm{dw})$ was one or two orders of magnitude higher than the values observed in previous studies reported by 
TABLE 1: The concentrations (means \pm SD) and contribution of PCBs discharged by the largest dyeing chemical industrial group entering the WWTP.

\begin{tabular}{|c|c|c|c|c|}
\hline PCBs (pg/L) & $\begin{array}{c}\text { Mother liquor of } \\
\text { manufacture }\end{array}$ & $\begin{array}{l}\text { Treated mother liquor } \\
\text { (entering the WWTP) }\end{array}$ & $\begin{array}{l}\text { Raw wastewater in } \\
\text { the WWTP }\end{array}$ & $\begin{array}{l}\text { Contribution rate to the } \\
\text { WWTP by the group (\%) }\end{array}$ \\
\hline \multicolumn{5}{|c|}{ Indicator PCB congener } \\
\hline PCB-28 & $3482.8 \pm 764.1$ & $242.3 \pm 96.2$ & $173.5 \pm 76.4$ & $11.2 \%$ \\
\hline PCB-52 & $162.74 \pm 46.1$ & $180.7 \pm 45.9$ & $249.1 \pm 32.5$ & $5.8 \%$ \\
\hline PCB-101 & $6.5 \pm 2.7$ & $4.1 \pm 2.8$ & $5.3 \pm 1.8$ & $6.2 \%$ \\
\hline PCB-118 & $96.26 \pm 30.3$ & $26.8 \pm 14.4$ & $48.7 \pm 22.6$ & $4.4 \%$ \\
\hline PCB-138 & $61.18 \pm 18.5$ & $177.9 \pm 46.7$ & $61.5 \pm 21.2$ & $23.1 \%$ \\
\hline PCB-153 & $85.62 \pm 26.4$ & $203.2 \pm 35.4$ & $89 \pm 25.4$ & $18.3 \%$ \\
\hline PCB-180 & $8.78 \pm 3.7$ & ND & $3.1 \pm 0.8$ & - \\
\hline Sum indicator PCBs & $3903.9 \pm 680.8$ & $835 \pm 241.4$ & $630.2 \pm 64.9$ & $10.7 \%$ \\
\hline \multicolumn{5}{|c|}{ Dioxin-like PCB congener } \\
\hline PCB-77 & $5897.46 \pm 1004.1$ & $212.9 \pm 63.6$ & $114.4 \pm 42.2$ & $14.9 \%$ \\
\hline PCB-81 & $2.7 \pm 0.85$ & $0.26 \pm 0.2$ & $0.13 \pm 0.1$ & $16.0 \%$ \\
\hline PCB-105 & $15.32 \pm 6.8$ & $8.1 \pm 2.8$ & $18.6 \pm 7.1$ & $3.5 \%$ \\
\hline PCB-114 & $5.7 \pm 3.4$ & $0.8 \pm 0.14$ & $0.45 \pm 0.1$ & $14.2 \%$ \\
\hline PCB-123 & $11.1 \pm 6.4$ & $4.4 \pm 1.4$ & $7.9 \pm 1.4$ & $4.5 \%$ \\
\hline PCB-126 & $2.04 \pm 2.4$ & $1.5 \pm 1.4$ & $1.9 \pm 1.4$ & $6.3 \%$ \\
\hline PCB-156 & $5 \pm 6.1$ & $8.7 \pm 2.8$ & $4.8 \pm 1.4$ & $14.5 \%$ \\
\hline PCB-167 & $18.4 \pm 17.2$ & $13 \pm 5.6$ & $13.8 \pm 4.2$ & $7.5 \%$ \\
\hline PCB-169 & $9.06 \pm 5.2$ & $0.6 \pm 0.6$ & $0.35 \pm 0.3$ & $13.7 \%$ \\
\hline PCB-189 & $1.68 \pm 1.4$ & $1.9 \pm 2.0$ & $0.4 \pm 0.3$ & $38.0 \%$ \\
\hline Sum DL-PCBs & $5968.5 \pm 995.3$ & $252.2 \pm 58$ & $162.7 \pm 33.3$ & $12.4 \%$ \\
\hline \multicolumn{5}{|c|}{ Other PCB congener } \\
\hline PCB-11 & $24870.2 \pm 14707$ & $18210.7 \pm 7636$ & $10321.6 \pm 763$ & $14.1 \%$ \\
\hline PCB-15 & $1398.8 \pm 240.4$ & $607.5 \pm 233$ & $241.6 \pm 84.8$ & $20.1 \%$ \\
\hline PCB-19 & $25.56 \pm 17.0$ & $9.4 \pm 9.9$ & $7.1 \pm 7.1$ & $10.6 \%$ \\
\hline Mono-CBs & $9621.5 \pm 4610.3$ & $892.4 \pm 240$ & $831.4 \pm 84.8$ & $8.6 \%$ \\
\hline Di-CBs & $32599.6 \pm 20265$ & $23518 \pm 9758$ & $12233.10 \pm 1004$ & $15.4 \%$ \\
\hline Tri-CBs & $6900.54 \pm 1517.4$ & $467.5 \pm 125$ & $636.60 \pm 169$ & $5.9 \%$ \\
\hline Tetra-CBs & $20411.54 \pm 10449$ & $627.9 \pm 132$ & $957.50 \pm 141$ & $5.2 \%$ \\
\hline Penta-CBs & $344.26 \pm 118.8$ & $84.2 \pm 32.5$ & $202.50 \pm 84.8$ & $3.3 \%$ \\
\hline Hexa-CBs & $671.42 \pm 138.6$ & $651.8 \pm 77.8$ & $342.10 \pm 113$ & $15.2 \%$ \\
\hline Hepta-CBs & $78.7 \pm 14.9$ & $171.4 \pm 29.7$ & $221.30 \pm 21.2$ & $6.2 \%$ \\
\hline Octa-CBs & $219.16 \pm 22.2$ & $75.2 \pm 32.5$ & $125.60 \pm 21.2$ & $4.8 \%$ \\
\hline Nona-CBs & $67.08 \pm 32.8$ & $58 \pm 19.8$ & $40.10 \pm 32.5$ & $11.6 \%$ \\
\hline Deca-CBs & $25.22 \pm 18.4$ & $13.3 \pm 8.5$ & $27 \pm 18.4$ & $3.9 \%$ \\
\hline$\sum 209$ PCBs & $70939.02 \pm 15635$ & $26559.7 \pm 9123$ & $15617.2 \pm 1691$ & $13.6 \%$ \\
\hline
\end{tabular}

Flow rate of the treated mother liquor: $8,000 \mathrm{~m}^{3} / \mathrm{d}$; flow rate of raw wastewater in the WWTP: $100,000 \mathrm{~m}^{3} / \mathrm{d}$; ND, not detected.

Blanchard et al., Guo et al., and Katsoyiannis and Samara $[12,16,22]$. According to the Chinese regulated law (GB189182002), PCB levels should be less than $200 \mathrm{ng} / \mathrm{g} \mathrm{dw}$ for agricultural use [26]. Therefore, the PCB levels in the WWTP sludge here were much higher than the maximum permissible concentration and were not suitable for agricultural purposes.

3.4. Phase Distribution of PCBs in Wastewater and Sludge of the WWTP. The distribution of single compounds varied among different systems, which might be attributed to several factors, such as the solute concentration, the amount of solids available for sorption, and the competition between pollutants for sorption sites of the particles [7, 27]. Seen from Figure 2, Di-CBs (78.3-93.1\%) were the predominant isomers in wastewater samples, followed by Tri-CBs (2.9$7.9 \%$ ) and Tetra-CBs (2.2-5.9\%). The proportion of other highly chlorinated biphenyls (Hexa-CBs to Deca-CBs) was less than $5 \%$ in each stage effluent. Similar to the profiles of PCB isomers in wastewater, Di-CBs (70.4-84.0\%) were the dominant isomers in sludge, followed by Mono-CBs (7.9-15.2\%) and Tri-CBs (5.4-16.8\%), with less than 3\% of contribution of other PCBs isomers. 
TABLE 2: The concentrations (means \pm SD) of PCBs in wastewater of each treatment stage at the WWTP.

\begin{tabular}{|c|c|c|c|c|c|}
\hline PCBs (pg/L) & RW & PSE & AHE & SSE & $\mathrm{HCE}$ \\
\hline \multicolumn{6}{|c|}{ Indicator PCB congener } \\
\hline PCB-28 & $173.5 \pm 76.4$ & $163.2 \pm 63.6$ & $603.3 \pm 56.6$ & $237.4 \pm 60.8$ & $93.9 \pm 49.5$ \\
\hline PCB-52 & $249.1 \pm 32.5$ & $131.2 \pm 32.5$ & $178.1 \pm 28.3$ & $54.3 \pm 25.4$ & $31.6 \pm 21.2$ \\
\hline PCB-101 & $5.3 \pm 1.8$ & $5.3 \pm 1.7$ & $2.1 \pm 1.4$ & $1.6 \pm 1.4$ & $1.1 \pm 0.7$ \\
\hline PCB-118 & $48.7 \pm 22.6$ & $24.7 \pm 8.5$ & $29.6 \pm 7.1$ & $8.1 \pm 5.6$ & $6 \pm 4.2$ \\
\hline PCB-138 & $61.5 \pm 21.2$ & $34.5 \pm 14.1$ & $18.4 \pm 7.2$ & $10.7 \pm 7.1$ & $6.1 \pm 4.2$ \\
\hline PCB-153 & $89 \pm 25.4$ & $55.8 \pm 22.1$ & $39 \pm 14.1$ & $12.9 \pm 8.5$ & $8.6 \pm 3.4$ \\
\hline PCB-180 & $3.1 \pm 0.8$ & $2 \pm 1.4$ & $2.5 \pm 1.4$ & $2.3 \pm 1.4$ & ND \\
\hline Sum indicator PCBs & $630.2 \pm 64.9$ & $416.7 \pm 35.6$ & $873 \pm 39.6$ & $327.3 \pm 79.2$ & $147.3 \pm 75.7$ \\
\hline \multicolumn{6}{|c|}{ Dioxin-like PCB congener } \\
\hline PCB-77 & $114.4 \pm 42.2$ & $93.7 \pm 33.9$ & $142.7 \pm 32.5$ & $52.2 \pm 28.3$ & $8.8 \pm 5.6$ \\
\hline PCB-81 & $0.13 \pm 0.1$ & $4.1 \pm 1.4$ & $3.9 \pm 1.6$ & ND & ND \\
\hline PCB-105 & $18.6 \pm 7.1$ & $10.9 \pm 5.6$ & $13.3 \pm 4.2$ & $3 \pm 2.8$ & $1.7 \pm 1.4$ \\
\hline PCB-114 & $0.45 \pm 0.1$ & $3.1 \pm 1.3$ & $2.2 \pm 1.3$ & $1.7 \pm 1.4$ & $1.4 \pm 1.4$ \\
\hline PCB-123 & $7.9 \pm 1.4$ & $2.9 \pm 1.4$ & $4.7 \pm 1.4$ & $3.9 \pm 1.4$ & $1.3 \pm 1.4$ \\
\hline PCB-126 & $1.9 \pm 1.4$ & ND & ND & ND & ND \\
\hline PCB-156 & $4.8 \pm 1.4$ & $4.8 \pm 1.1$ & $7.1 \pm 2.5$ & $1.5 \pm 1.4$ & $1.3 \pm 1.2$ \\
\hline PCB-167 & $13.8 \pm 4.2$ & $7.3 \pm 4.2$ & $6.4 \pm 1.8$ & $6.1 \pm 2.8$ & $1.1 \pm 0.7$ \\
\hline PCB-169 & $0.35 \pm 0.3$ & ND & ND & ND & ND \\
\hline PCB-189 & $0.4 \pm 0.3$ & ND & ND & ND & ND \\
\hline Sum DL-PCBs & $162.7 \pm 33.3$ & $126.8 \pm 29.4$ & $180.3 \pm 29.0$ & $68.4 \pm 26.9$ & $15.6 \pm 3.5$ \\
\hline \multicolumn{6}{|c|}{ Other PCB congener } \\
\hline PCB-11 & $10321.6 \pm 763$ & $10191.4 \pm 552$ & $9931 \pm 495$ & $9944.3 \pm 424$ & $9479.1 \pm 424$ \\
\hline PCB-15 & $241.6 \pm 84.8$ & $105 \pm 51$ & $76.5 \pm 42.4$ & $31.2 \pm 35.4$ & $10.3 \pm 8.5$ \\
\hline PCB-19 & $7.1 \pm 7.1$ & $4.9 \pm 4.2$ & $4.6 \pm 2.8$ & $4.2 \pm 2.8$ & $3.2 \pm 2.8$ \\
\hline Mono-CBs & $831.4 \pm 84.8$ & $642.7 \pm 63.6$ & $512.4 \pm 70.7$ & $87.5 \pm 70.7$ & $43.6 \pm 35.4$ \\
\hline Di-CBs & $12233.10 \pm 1004$ & $12186.4 \pm 992$ & $11804.2 \pm 735$ & $11426.4 \pm 665$ & $11160.9 \pm 566$ \\
\hline Tri-CBs & $636.60 \pm 169$ & $425.3 \pm 127$ & $1182.2 \pm 282$ & $707.9 \pm 254$ & $348.6 \pm 212$ \\
\hline Tetra-CBs & $957.50 \pm 141$ & $554.3 \pm 141$ & $879.5 \pm 141$ & $302.9 \pm 127$ & $261.3 \pm 107$ \\
\hline Penta-CBs & $202.50 \pm 84.8$ & $158.9 \pm 73.5$ & $124.4 \pm 73.5$ & $74.1 \pm 73.5$ & $36.8 \pm 31.3$ \\
\hline Hexa-CBs & $342.10 \pm 113$ & $197.4 \pm 113$ & $152.7 \pm 113$ & $54.9 \pm 28.3$ & $27.1 \pm 22.6$ \\
\hline Hepta-CBs & $221.30 \pm 21.2$ & $121.4 \pm 21.2$ & $107.3 \pm 21.2$ & $67.3 \pm 48.1$ & $32.4 \pm 32.5$ \\
\hline Octa-CBs & $125.60 \pm 21.2$ & $191.9 \pm 99$ & $165.7 \pm 84.8$ & $89.6 \pm 70.7$ & $37.6 \pm 21.2$ \\
\hline Nona-CBs & $40.10 \pm 32.5$ & $46.1 \pm 22.6$ & $44.1 \pm 19.8$ & $58.3 \pm 19.8$ & $31.8 \pm 14.1$ \\
\hline Deca-CBs & $27 \pm 18.4$ & $16.5 \pm 11.3$ & $19.5 \pm 8.5$ & $16.8 \pm 8.5$ & $8.2 \pm 8.5$ \\
\hline$\sum 209$ PCBs & $15617.2 \pm 1691$ & $14540.9 \pm 1397$ & $14992 \pm 618$ & $12885.7 \pm 659$ & $11988.3 \pm 510$ \\
\hline SS (mg/L) & 450 & 120 & 100 & 30 & 12 \\
\hline
\end{tabular}

RW: raw wastewater (mixing influent); PSE: primary sedimentation effluent; AHE: anaerobic hydrolysis effluent; SSE: secondary sedimentation effluent; HCE: high-density clarifier effluent; and ND: not detected.

The partitioning behaviors of organic chemicals are essential to their fate in the WWTP, as dissolved and adsorbed phases are differently available to different fate processes [11]. Based on the PCBs concentrations in liquidsolid phase, water quantity, and SPM yield, the relative distribution of PCB isomers between the dissolved and adsorbed phases was obtained in the present study. Figure 3 presents the proportions of adsorbed PCB isomers in the influent and effluents of each treatment stage. It is clear that the adsorbed fraction of $\sum 209$ PCBs was found to range from $89.8 \%$ to $97.4 \%$ in the influent and effluent of each treatment stage, much higher than the findings of
Katsoyiannis and Samara [28] with less than $60 \%$ of adsorbed $\sum$ PCBs in the influent of WWTP in Thessaloniki, Greece. The adsorbed PCB isomers in secondary sedimentation effluent were absolutely predominant, varying between $87.3 \%$ for Octa-CBs and $99.8 \%$ for Mono-CBs. Likewise, significant adsorbed fractions of PCB isomers were also found in the raw wastewater, primary sedimentation effluent, and anaerobic hydrolysis effluent, varying between $53.4 \%$ for Nona-CBs and 99.1\% for Tri-CBs, $54.0 \%$ for Nona-CBs and $99.4 \%$ for TriCBs and $45.9 \%$ for Nona-CBs and $98.3 \%$ for Mono-CBs, respectively. Differently, in high-density clarifier effluent, Nona-CBs and Deca-CBs were more "dissolved" among 
TABLE 3: The concentrations (means \pm SD) of PCBs in SPM and sludge of each treatment stage at the WWTP.

\begin{tabular}{|c|c|c|c|c|c|c|}
\hline $\begin{array}{l}\text { PCBs } \\
\text { (ng/g, dw) }\end{array}$ & SPM in $\mathrm{RW}$ & PSS & SPM in AHE & AS & HCS & FS \\
\hline \multicolumn{7}{|c|}{ Indicator PCB congener } \\
\hline PCB-28 & $100.5 \pm 21.2$ & $266.6 \pm 63.6$ & $316.7 \pm 82$ & $255.8 \pm 89.1$ & $87.36 \pm 32.5$ & $1317.1 \pm 158$ \\
\hline PCB-52 & $6.289 \pm 2.83$ & $17.3 \pm 7.1$ & $19.75 \pm 7.07$ & $22.41 \pm 9.90$ & $7.85 \pm 1.74$ & $26.4 \pm 15.1$ \\
\hline PCB-101 & $0.139 \pm 0.04$ & $0.357 \pm 0.04$ & $0.437 \pm 0.07$ & $0.377 \pm 0.09$ & $1 \pm 0.042$ & $3.101 \pm 0.17$ \\
\hline PCB-118 & $0.416 \pm 0.07$ & $1.17 \pm 0.08$ & $1.365 \pm 0.11$ & $1.665 \pm 0.14$ & $0.204 \pm 0.08$ & $2.763 \pm 0.29$ \\
\hline PCB-138 & $0.243 \pm 0.05$ & $0.698 \pm 0.06$ & $0.869 \pm 0.14$ & $0.903 \pm 0.32$ & $0.29 \pm 0.06$ & $3.002 \pm 0.51$ \\
\hline PCB-153 & $0.394 \pm 0.06$ & $1.013 \pm 0.07$ & $1.362 \pm 0.31$ & $1.829 \pm 0.49$ & $0.307 \pm 0.07$ & $2.893 \pm 0.73$ \\
\hline PCB-180 & $0.032 \pm 0.01$ & $0.063 \pm 0.03$ & $0.087 \pm 0.07$ & $0.108 \pm 0.12$ & $0.033 \pm 0.03$ & $0.282 \pm 0.20$ \\
\hline Sum Ind & $108.0 \pm 18.5$ & $287.2 \pm 56.7$ & $340.5 \pm 89.8$ & $283.1 \pm 100$ & $97.05 \pm 34.5$ & $1355.6 \pm 175$ \\
\hline \multicolumn{7}{|c|}{ Dioxin-like PCB congener } \\
\hline PCB-77 & $4.835 \pm 0.57$ & $12.84 \pm 6.22$ & $14.81 \pm 7.14$ & $15.02 \pm 8.63$ & $4.85 \pm 2.02$ & $20.15 \pm 13.8$ \\
\hline PCB-81 & $0.027 \pm 0.01$ & $0.043 \pm 0.03$ & $0.057 \pm 0.04$ & $0.064 \pm 0.06$ & $0.02 \pm 0.01$ & $0.119 \pm 0.09$ \\
\hline PCB-105 & $0.137 \pm 0.03$ & $0.404 \pm 0.05$ & $0.463 \pm 0.07$ & $0.519 \pm 0.09$ & $0.119 \pm 0.05$ & $1.345 \pm 0.18$ \\
\hline PCB-114 & $0.01 \pm 0.004$ & $3.1 \pm 1.3$ & $0.026 \pm 0.01$ & $0.076 \pm 0.04$ & $0.015 \pm 0.01$ & $0.112 \pm 0.08$ \\
\hline PCB-123 & $0.002 \pm 0.001$ & $2.9 \pm 1.4$ & $0.014 \pm 0.01$ & $0.08 \pm 0.01$ & $0.064 \pm 0.004$ & $0.368 \pm 0.19$ \\
\hline PCB-126 & ND & ND & $0.012 \pm 0.01$ & $0.033 \pm 0.03$ & $0.012 \pm 0.001$ & $0.242 \pm 0.04$ \\
\hline PCB-156 & ND & $4.8 \pm 1.1$ & $0.014 \pm 0.01$ & $0.095 \pm 0.04$ & $0.026 \pm 0.001$ & $0.859 \pm 0.06$ \\
\hline PCB-167 & $0.204 \pm 0.006$ & $7.3 \pm 4.2$ & $0.643 \pm 0.05$ & $0.496 \pm 0.06$ & $0.352 \pm 0.02$ & $0.648 \pm 0.11$ \\
\hline PCB-169 & ND & ND & $0.042 \pm 0.02$ & $0.086 \pm 0.04$ & $0.025 \pm 0.034$ & $0.103 \pm 0.10$ \\
\hline PCB-189 & $0.038 \pm 0.008$ & ND & $0.101 \pm 0.06$ & $0.173 \pm 0.09$ & $0.105 \pm 0.047$ & $0.257 \pm 0.18$ \\
\hline Sum DL & $5.254 \pm 0.55$ & $126.8 \pm 29.4$ & $16.19 \pm 7.44$ & $16.64 \pm 8.83$ & $5.59 \pm 2.207$ & $24.2 \pm 14.7$ \\
\hline \multicolumn{7}{|c|}{ Other PCB congener } \\
\hline PCB-11 & $803.23 \pm 212$ & $10191.4 \pm 552$ & $2702.4 \pm 655$ & $3066.8 \pm 744$ & $1135.5 \pm 459$ & $4478.4 \pm 842$ \\
\hline PCB-15 & ND & $105 \pm 51$ & $0.164 \pm 0.02$ & $0.217 \pm 0.03$ & $0.352 \pm 0.11$ & $0.235 \pm 0.12$ \\
\hline PCB-19 & ND & $4.9 \pm 4.2$ & $0.013 \pm 0.01$ & $0.025 \pm 0.01$ & $0.083 \pm 0.037$ & $0.038 \pm 0.04$ \\
\hline Mono-CBs & $95.47 \pm 35.35$ & $642.7 \pm 63.6$ & $306.3 \pm 61.6$ & $320.1 \pm 65.5$ & $284.6 \pm 45.9$ & $510 \pm 94.7$ \\
\hline Di-CBs & $1046.1 \pm 311$ & $12186 \pm 992$ & $2852.3 \pm 726$ & $3389.3 \pm 800$ & $1146.4 \pm 527$ & $4931 \pm 1128$ \\
\hline Tri-CBs & $160.57 \pm 46.67$ & $425.3 \pm 127$ & $497.3 \pm 109$ & $219.5 \pm 109$ & $102.3 \pm 51.2$ & $862.4 \pm 143$ \\
\hline Tetra-CBs & $17.151 \pm 7.07$ & $554.3 \pm 141$ & $51.62 \pm 25.3$ & $62.95 \pm 27.9$ & $24.17 \pm 14.7$ & $78.82 \pm 36.2$ \\
\hline Penta-CBs & $1.875 \pm 0.007$ & $158.9 \pm 73.5$ & $6.324 \pm 1.6$ & $10.87 \pm 3.34$ & $12.85 \pm 0.99$ & $6.886 \pm 3.68$ \\
\hline Hexa-CBs & $1.572 \pm 0.848$ & $197.4 \pm 113$ & $6.242 \pm 1.23$ & $8.965 \pm 1.67$ & $2.02 \pm 0.848$ & $12.31 \pm 2.14$ \\
\hline Hepta-CBs & $0.78 \pm 0.424$ & $121.4 \pm 21.2$ & $3.485 \pm 0.92$ & $6.758 \pm 1.51$ & $1.359 \pm 0.424$ & $11.63 \pm 1.65$ \\
\hline Octa-CBs & $0.587 \pm 0.283$ & $191.9 \pm 99$ & $2.436 \pm 0.61$ & $4.744 \pm 1.23$ & $1.262 \pm 0.28$ & $5.11 \pm 1.29$ \\
\hline Nona-CBs & $0.102 \pm 0.01$ & $46.1 \pm 22.6$ & $0.378 \pm 0.03$ & $7.057 \pm 1.89$ & $0.226 \pm 0.14$ & $8.77 \pm 1.73$ \\
\hline Deca-CBs & $0.213 \pm 0.007$ & $16.5 \pm 11.3$ & $0.984 \pm 0.55$ & $3.109 \pm 0.79$ & $0.082 \pm 0.31$ & $5.485 \pm 0.94$ \\
\hline$\sum 209$ PCBs & $1324.4 \pm 306.9$ & $14541 \pm 1397$ & $3727.4 \pm 927$ & $4033 \pm 1014$ & $1875.3 \pm 641$ & $6432 \pm 1123$ \\
\hline $\mathrm{SS}(\mathrm{mg} / \mathrm{L})$ & 450 & $1 * 10^{4}$ & 100 & $2 * 10^{3}$ & $1 * 10^{3}$ & $2 * 10^{4}$ \\
\hline
\end{tabular}

SPM: suspended particulate matter; RW: mixing influent (raw wastewater); PSS: primary sedimentation sludge; AHE: anaerobic hydrolysis effluent; AS: activated sludge; HCS: high-density clarifier sludge; FS: final sludge; ND: not detected; sum Ind.: sum indicator PCBs; and sum DL: sun DL-PCBs.

the PCB isomers, with only $30.0 \%$ and $39.2 \%$ in the solid phase, although the adsorbed fractions of other PCB isomers were all above $65 \%$ in this stage. Generally, better removal can be obtained for higher MW congeners by sorption onto particles or microbes owing to their high hydrophobicity [11]. But what was reason about this unusual result? As seen from Tables 2 and 3, the mean concentrations of dissolved NanoCBs and Deca-CBs were 31.8 and $8.2 \mathrm{pg} / \mathrm{L}$ in HCE and the average levels of adsorbed Nano-CBs and Deca-CBs were 0.226 and $0.082 \mathrm{ng} / \mathrm{g}$, respectively. On the one hand, maybe due to the very low concentrations of them in dissolved, (possible) oxidation by ferrate or flocculation/adsorption by $\mathrm{Fe}(\mathrm{OH})_{3}$ cannot occur accompanied with the competitive effect among various pollutants. On the other hand, the fraction remaining in the treated wastewater, unexpectedly high for hydrophobic compounds, could be explained by 


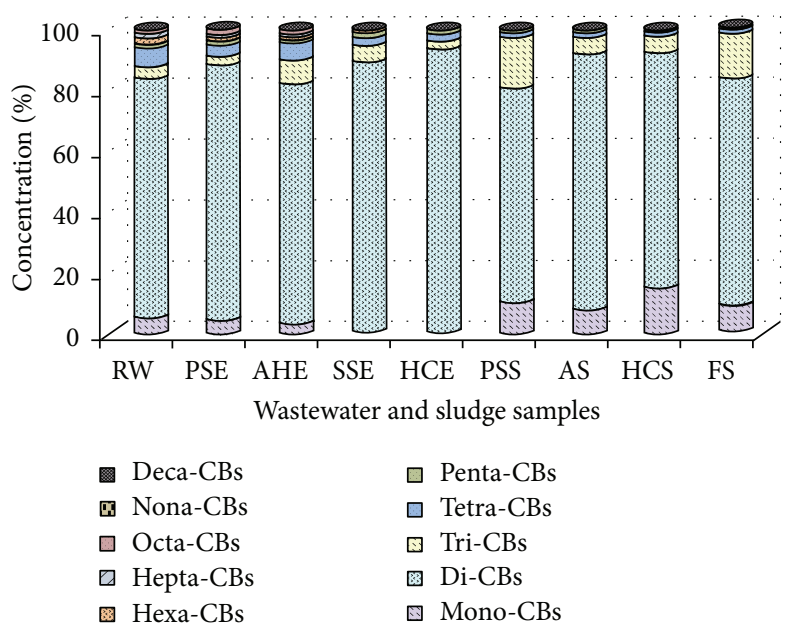

FIGURE 2: PCB isomer profiles in wastewater and sludge samples of the WWTP.

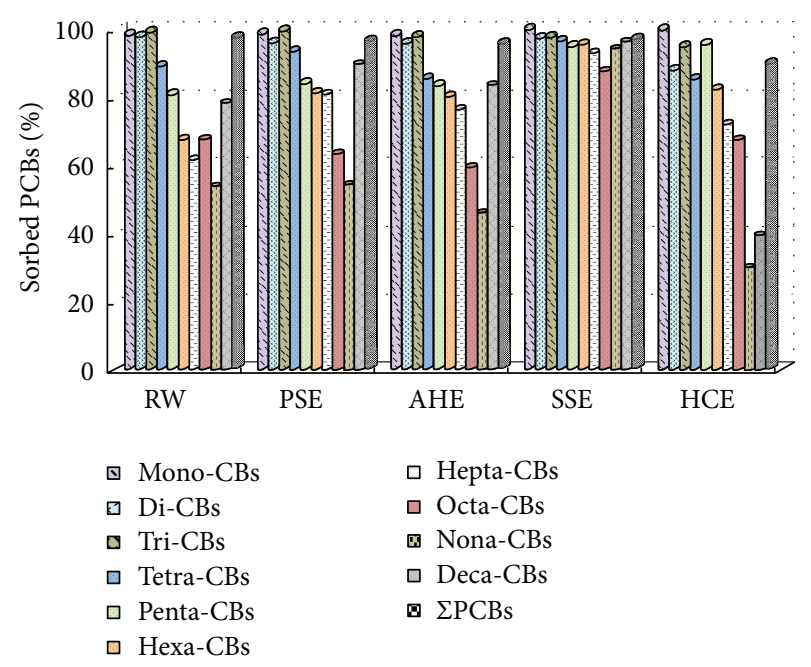

FIGURE 3: Distribution of PCBs in the adsorbed phase of the influent and effluent of each treatment stage.

possible adsorption of PCBs onto nonsettleable solids, which remain in the wastewater stream due to the lack of chemical coagulation [28].

3.5. Removal of PCBs throughout the Treatment Processes in the WWTP. Based on the mean concentrations of dissolved PCB isomers in each treatment stage, the mean removals can be calculated using the general equation:

$$
R(\%)=\frac{\left(C_{\text {in }}-C_{\text {out }}\right)}{C_{\text {in }}} \times 100,
$$

where $C_{\text {in }}$ and $C_{\text {out }}$ are the daily amounts of PCBs entering and exiting each treatment stage, respectively. The results are shown in Figure 4. Poor removal efficiencies were obtained in primary sedimentation and anaerobic hydrolysis stage, which may be due to the competitive adsorption between pollutants and the selective adsorption of PAC flocculants and anaerobic

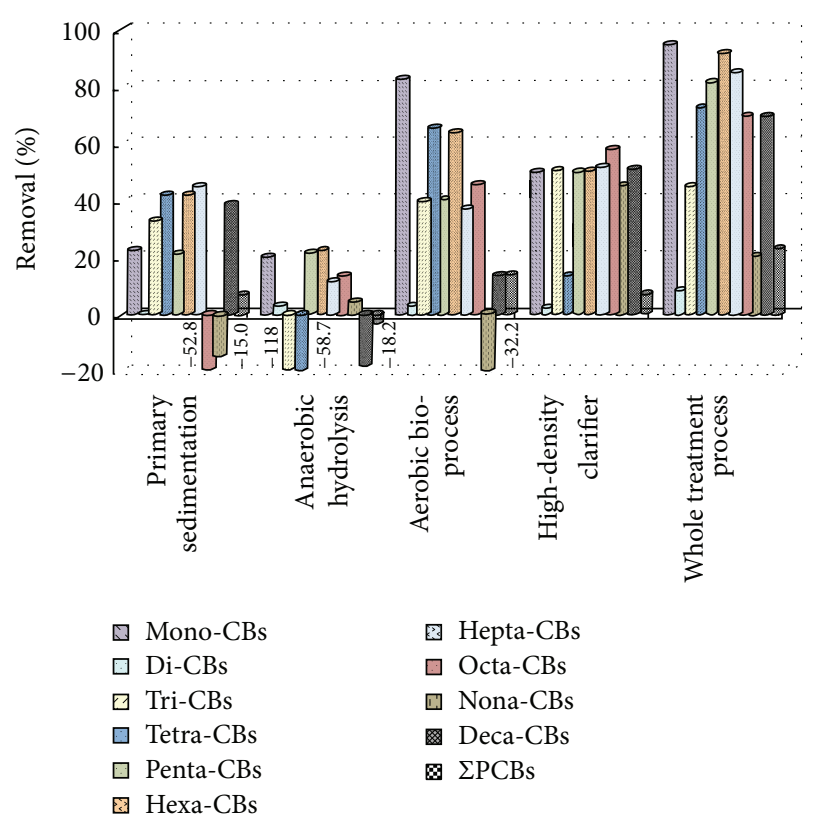

FIGURE 4: Mean removal percentages of dissolved PCB isomers during each treatment stage.

sludge. In the following treatment stages, removal efficiencies for most PCB isomers were around or above $40 \%$ except for Di-, Nona-, and Deca-CBs in aerobic bioprocess and Diand Tetra-CBs in high-density clarifier stage. Furthermore, interestingly, removal efficiencies of several PCB isomers (Octa-CBs and Nona-CBs in primary sedimentation, Tri-CBs and Tetra-CBs in anaerobic hydrolysis, etc.) were negative (below zero) as shown, which resulted from the surge of the PCBs in the effluents.

Throughout the whole treatment process, the total removal efficiency of $\sum 209$ PCBs was $23.2 \%$. In detail, the removal efficiencies of $\mathrm{Di}-\mathrm{CBs}$ and Nona-CBs were the lowest among all the isomers (8.8\% for Di-CBs and $20.7 \%$ for Nona-CBs). The removals of other PCB isomers were higher than those. Especially, for Mono-CBs and Hexa-CBs, the removal efficiencies were higher than $90 \%$ and high efficiencies of over $80 \%$ were achieved for Penta-CBs and Hepta-CBs. In comparison, the removals of PCBs were $20 \%-$ $91 \%$ in 26 WPCPs in New York and average removal were 77.3\% described by Durell and Lizotte [23]. Higher removal was found in the WWTPs in Norway with more than $90 \%$ removal [24]. This was because PCB concentrations were very low with $\mathrm{pg} / \mathrm{L}$ grade in the WWTP tested and the concentrations in other WWTPs were with ng/L grade. The lower the concentration of PCBs is, the more difficult their removal becomes.

Based on the results above and other published papers, the removals of hydrophobic chemicals in wastewater treatment system are strongly dependent on their sorptive behaviors $[8,12]$. The adsorption of the hydrophobic organic pollutants is generally affected by their octanol-water partition coefficients $\left(K_{\mathrm{ow}}\right)$. In order to verify whether these findings are applicable to the WWTPs in China, the removal 


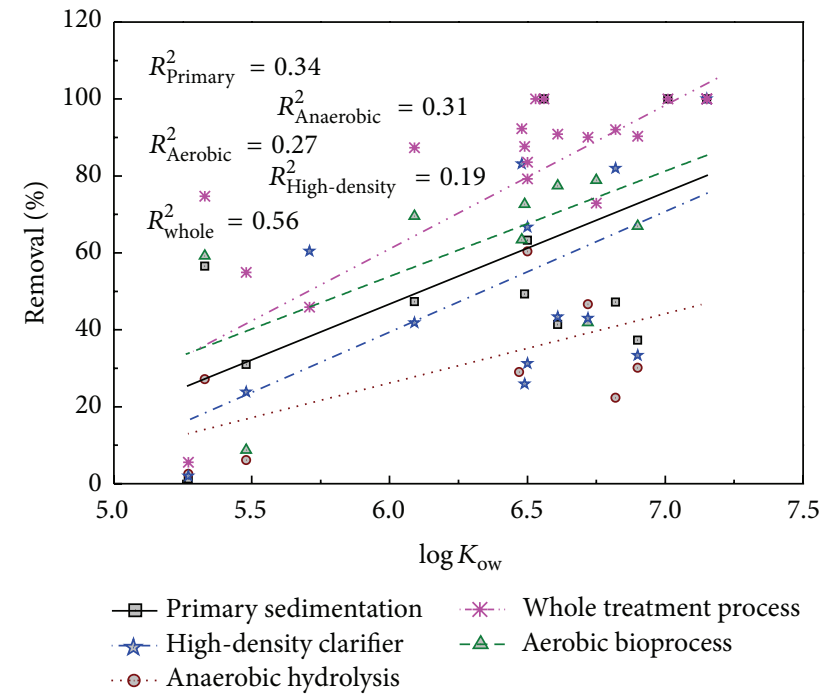

Figure 5: Percent removal of individual PCBs versus $\log K_{\text {ow }}$ in each treatment stage.

efficiency of each PCB congener is plotted against the $\log K_{\text {ow }}$ $[29,30]$, which is shown in Figure 5. There was a weak linear correlation $\left(R^{2}=0.56\right)$ between the removal of $\mathrm{PCB}$ congeners and their $\log K_{\text {ow }}$ values in the whole treatment process. However, a relatively poor linear relationship $\left(R^{2}\right.$ $0.19-0.34$ ) was observed in primary sedimentation, anaerobic hydrolysis, aerobic bioprocess, and high-density clarifier stage, which indicated that except for adsorption on sludge particles or microorganisms, other mechanisms, such as advection, volatilization, biotransformation, or (possible) oxidation and coagulation by ferrate might also be important for the less hydrophobic compounds of this class ( $\log K_{\text {ow }}$ $5.2-7.2)[12,31]$.

\section{Conclusions}

Dissolved and adsorbed concentrations of twenty PCB congeners and total PCBs (from Mono-CBs to Deca-CBs) were detected in each treatment stage in a centralized WWTP, located in a chemical industry zone in Zhejiang, China. It was investigated that the industrial activities, especially the dyeing chemical processes, were the main sources of PCBs entering the WWTP. The contribution of $\sum 209$ PCBs discharged by the largest dyeing chemical group to the WWTP was about $13.6 \%$.

PCBs entering the WWTP could be dissolved in the wastewater or adsorbed onto the particulates. The most abundant PCB was PCB-11 in the liquid and solid phase of each treatment stage, accounting for more than $60 \%$ of the total 209 PCBs. Meanwhile, the PCB levels in the WWTP sludge were much higher than the limit set by Chinese regulated laws. Thus, the sludge was not suitable for agricultural purposes. Partitioning behavior of PCBs between the dissolved and adsorbed phases suggested that Di-CBs were the most dominant isomers in the WWTP accounting for over $70 \%$ of $\sum 209$ PCBs. $89.8-97.4 \%$ of Di-CBs were mainly adsorbed on the particles and sludge in the influent and effluent of each treatment stage. The total removal efficiency of $\sum$ PCBs (209) was only $23.2 \%$ throughout the whole treatment process, although more than $80 \%$ of Mono$\mathrm{CBs}$, Penta-CBs, Hexa-CBs, and Hepta-CBs were removed. In each treatment stage, poor removal efficiencies were found in primary sedimentation and anaerobic hydrolysis, which was due to the difficult settlement and biodegradation of PCB-11. Furthermore, a weak linear correlation between the removal of $\mathrm{PCB}$ congeners and their $\log K_{\mathrm{ow}}$ values was found throughout the whole treatment process, which indicated that except for adsorption on sludge particles or microorganisms, other mechanisms, such as advection, volatilization, biotransformation, or oxidation and coagulation by ferrate might also be important for the less hydrophobic compounds of this class.

\section{Conflict of Interests}

The authors declare that there is no conflict of interests regarding this paper.

\section{Acknowledgments}

The authors would appreciate the financial support to this study provided by MOST Project of China (no. 2008BAC32B06) and NSFC of China (nos. 21076188, 20836008, 20976158, 20990221, and 21076189), and the Key Innovation Team for Science and Technology of Zhejiang Province of China (2009R50047). They are sincerely grateful to Professor Jiang Guibin and Zhang Qinghua in Research Center for Eco-Environmental Sciences, Chinese Academy of Sciences, Beijing for providing us with sample pretreatment and analytical method.

\section{References}

[1] K. C. Jones and P. de Voogt, "Persistent organic pollutants (POPs): state of the science," Environmental Pollution, vol. 100, no. 1-3, pp. 209-221, 1999.

[2] K. Breivik, A. Sweetman, J. M. Pacyna, and K. C. Jones, "Towards a global historical emission inventory for selected PCB congeners-a mass balance approach: 1. Global production and consumption," Science of the Total Environment, vol. 290, no. 1-3, pp. 181-198, 2002.

[3] L. A. Rodenburg, J. Guo, S. Du, and G. J. Cavallo, "Evidence for unique and ubiquitous environmental sources of 3,3/dichlorobiphenyl (PCB 11)," Environmental Science and Technology, vol. 44, no. 8, pp. 2816-2821, 2010.

[4] B. Fraser, "Researchers find little-known PCB 'pretty much everywhere," Environmental Science and Technology, vol. 44, no. 8, pp. 2753-2754, 2010.

[5] M. Pandelova, R. Piccinelli, S. Kasham, B. Henkelmann, C. Leclercq, and K. W. Schramm, "Assessment of dietary exposure to PCDD/F and dioxin-like PCB in infant formulae available on the EU market," Chemosphere, vol. 81, no. 8, pp. 1018-1021, 2010.

[6] F. Samara, C. W. Tsai, and D. S. Aga, "Determination of potential sources of PCBs and PBDEs in sediments of the Niagara River," Environmental Pollution, vol. 139, no. 3, pp. 489-497, 2006. 
[7] A. Martinez, K. Wang, and K. C. Hornbuckle, "Fate of PCB congeners in an industrial harbor of lake Michigan," Environmental Science \& Technology, vol. 44, no. 8, pp. 2803-2808, 2010.

[8] T. Pham and S. Proulx, "PCBs and PAHs in the Montreal urban community (Quebec, Canada) wastewater treatment plant and in the effluent plume in the St Lawrence river," Water Research, vol. 31, no. 8, pp. 1887-1896, 1997.

[9] Y. W. Wang, X. M. Li, A. Li et al., "Effect of municipal sewage treatment plant effluent on bioaccumulation of polychlorinated biphenyls and polybrominated diphenyl ethers in the recipient water," Environmental Science \& Technology, vol. 41, no. 17, pp. 6026-6032, 2007.

[10] P.-A. Bergqvist, L. Augulyte, and V. Jurjoniene, "PAH and PCB removal efficiencies in Umeå (Sweden) and Šiauliai (Lithuania) municipal wastewater treatment plants," Water, Air, and Soil Pollution, vol. 175, no. 1-4, pp. 291-303, 2006.

[11] G. Byrns, "The fate of xenobiotic organic compounds in wastewater treatment plants," Water Research, vol. 35, no. 10, pp. 2523-2533, 2001.

[12] A. Katsoyiannis and C. Samara, "Persistent organic pollutants (POPs) in the sewage treatment plant of Thessaloniki, Northern Greece: occurrence and removal," Water Research, vol. 38, no. 11, pp. 2685-2698, 2004.

[13] H. Liu, Q. Zhang, Z. Cai, A. Li, Y. Wang, and G. Jiang, "Separation of polybrominated diphenyl ethers, polychlorinated biphenyls, polychlorinated dibenzo-p-dioxins and dibenzofurans in environmental samples using silica gel and florisil fractionation chromatography," Analytica Chimica Acta, vol. 557, no. 1-2, pp. 314-320, 2006.

[14] US EPA, "Method 1668 Revision A: Chlorinated biphenyl congeners in water, soil, sediment, and tissue by HRGC/HRMS," Washington, DC, USA, EPA No. EPA-821-R-00-002, 1999.

[15] R. Yang, Y. Wang, A. Li et al., "Organochlorine pesticides and PCBs in fish from lakes of the Tibetan Plateau and the implications," Environmental Pollution, vol. 158, no. 6, pp. 23102316, 2010.

[16] L. Guo, B. Zhang, K. Xiao, Q. H. Zheng, and M. H. Zheng, "Levels and distributions of polychlorinated biphenyls in sewage sludge of urban wastewater treatment plants," Journal of Environmental Sciences, vol. 21, no. 4, pp. 468-473, 2009.

[17] S. Litten, B. Fowler, and D. Luszniak, "Identification of a novel PCB source through analysis of 209 PCB congeners by US EPA modified method 1668," Chemosphere, vol. 46, no. 9-10, pp. 1457-1459, 2002.

[18] D. Hu and K. C. Hornbuckle, "Inadvertent polychlorinated biphenyls in commercial paint pigments," Environmental Science and Technology, vol. 44, no. 8, pp. 2822-2827, 2010.

[19] F. Rajaei, A. Esmaili-Sari, N. Bahramifar, M. Ghasempouri, and M. Savabieasfahani, "Avian liver organochlorine and PCB from South coast of the Caspian Sea, Iran," Ecotoxicology, vol. 19, no. 2, pp. 329-337, 2010.

[20] W. Herbst and K. Hunger, Industrial Organic Pigments, WileyVCH, Weinheim, Germany, 3rd edition, 2004.

[21] H. L. He, Fine Chemicals Daquan: Dyes (Chinese Version), Zhejiang Science and Technology Press, Hangzhou, China, 2000.

[22] M. Blanchard, M. J. Teil, D. Ollivon, L. Legenti, and M. Chevreuil, "Polycyclic aromatic hydrocarbons and polychlorobiphenyls in wastewaters and sewage sludges from the Paris area (France)," Environmental Research, vol. 95, no. 2, pp. 184-197, 2004.
[23] G. S. Durell and R. D. Lizotte Jr., "PCB levels at 26 New York City and New Jersey WPCPs that discharge to the New York/New Jersey Harbor Estuary," Environmental Science \& Technology, vol. 32, no. 8, pp. 1022-1031, 1998.

[24] C. Vogelsang, M. Grung, T. G. Jantsch, K. E. Tollefsen, and H. Liltved, "Occurrence and removal of selected organic micropollutants at mechanical, chemical and advanced wastewater treatment plants in Norway," Water Research, vol. 40, no. 19, pp. 3559-3570, 2006.

[25] S. J. de Luca, M. Cantelli, and M. A. de Luca, "Ferrate vs traditional coagulants in the treatment of combined industrial wastes," Water Science and Technology, vol. 26, no. 9-11, pp. 2077-2080, 1992.

[26] Ministry of Environmental Protection of the People's Republic of China, "Discharge standard of pollutants for municipal wastewater treatment plant (GB 18918-2002)".

[27] S. Morris and J. N. Lester, "Behaviour and fate of polychlorinated biphenyls in a pilot wastewater treatment plant," Water Research, vol. 28, no. 7, pp. 1553-1561, 1994.

[28] A. Katsoyiannis and C. Samara, "Persistent organic pollutants (POPs) in the conventional activated sludge treatment process: fate and mass balance," Environmental Research, vol. 97, no. 3, pp. 245-257, 2005.

[29] D. W. Hawker and D. W. Connell, "Octanol-water partition coefficients of polychlorinated biphenyl congeners," Environmental Science and Technology, vol. 22, no. 4, pp. 382-387, 1988.

[30] M. F. Yeh and C. S. Hong, "Octanol-water partition coefficients of non-ortho- and mono-ortho-substituted polychlorinated biphenyls," Journal of Chemical \& Engineering Data, vol. 47, no. 2, pp. 209-215, 2002.

[31] T. D. Waite and K. A. Gray, "Oxidation and coagulation of wastewater effluent utilizing ferrate (VI) ion," Studies in Environmental Science, vol. 23, pp. 407-420, 1984. 

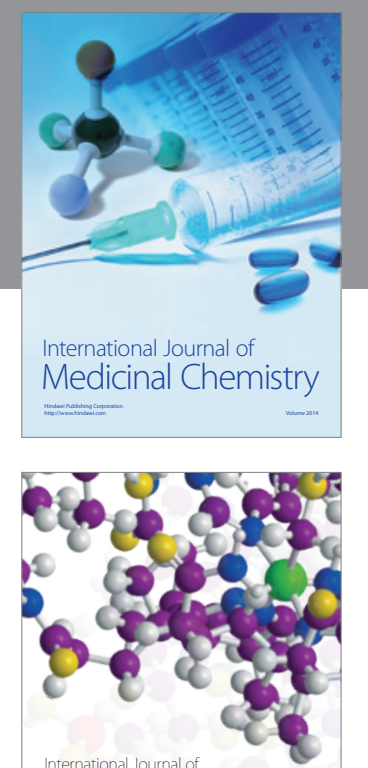

\section{Carbohydrate} Chemistry

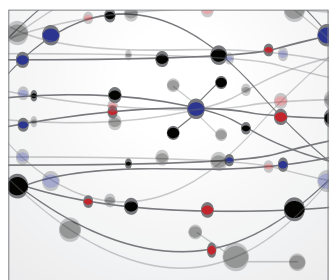

The Scientific World Journal
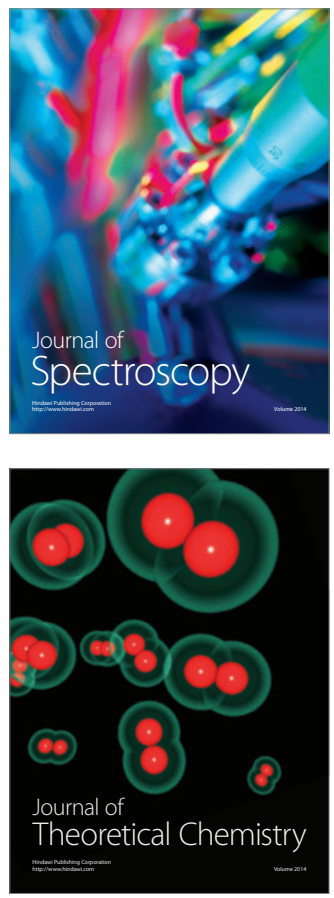
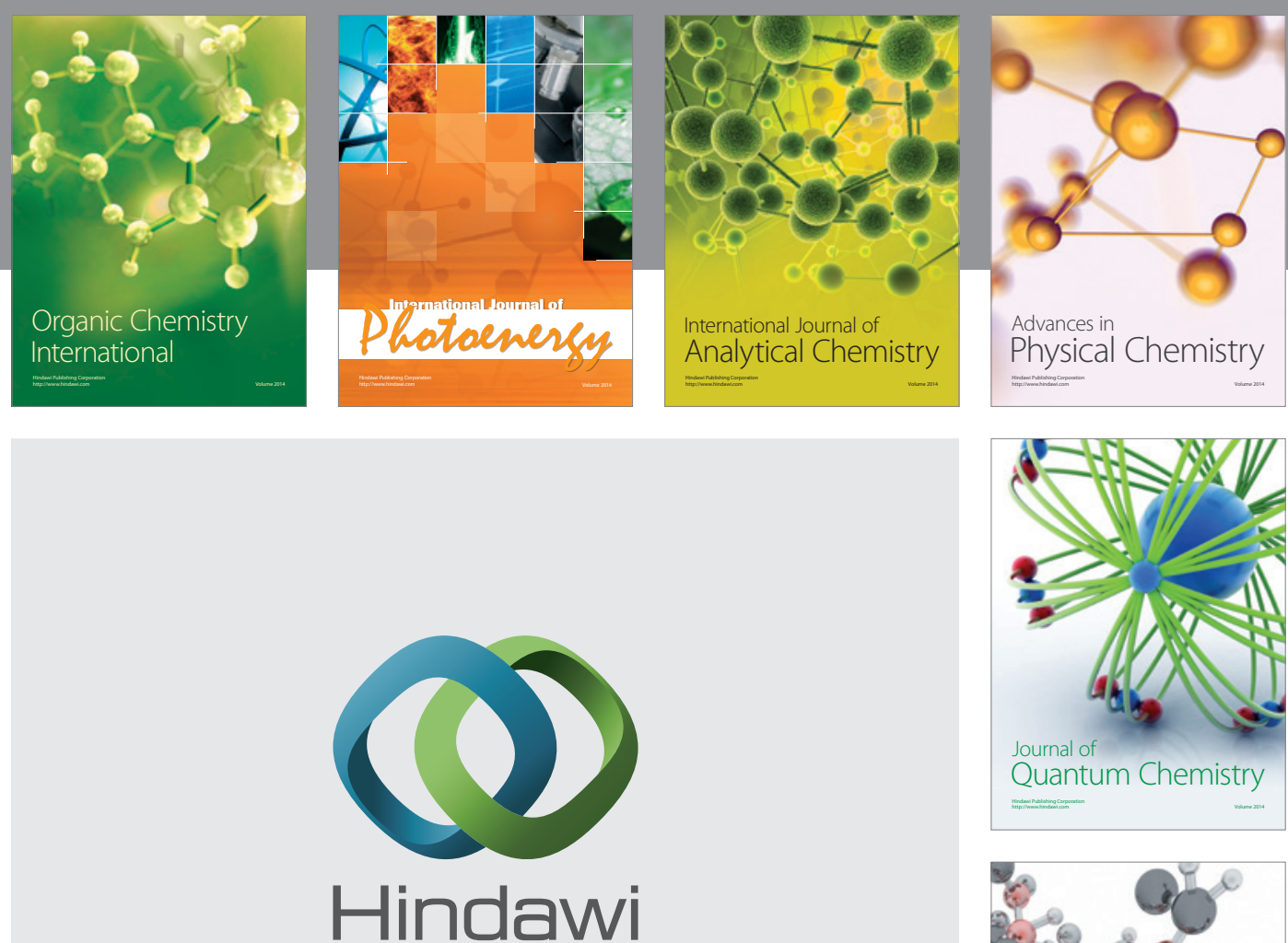

Submit your manuscripts at

http://www.hindawi.com

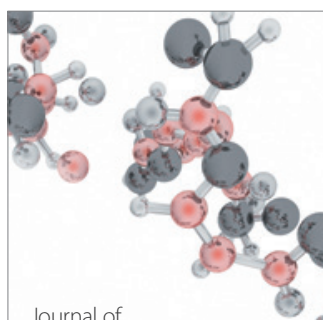

Analytical Methods

in Chemistry

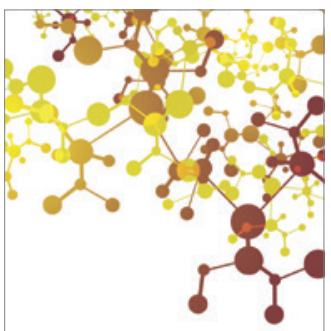

Journal of

Applied Chemistry

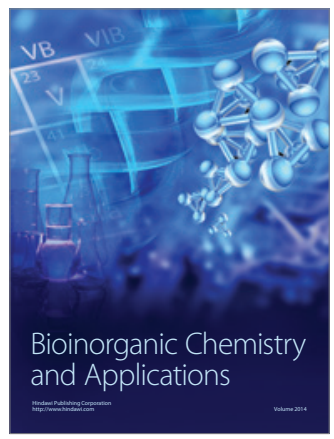

Inorganic Chemistry
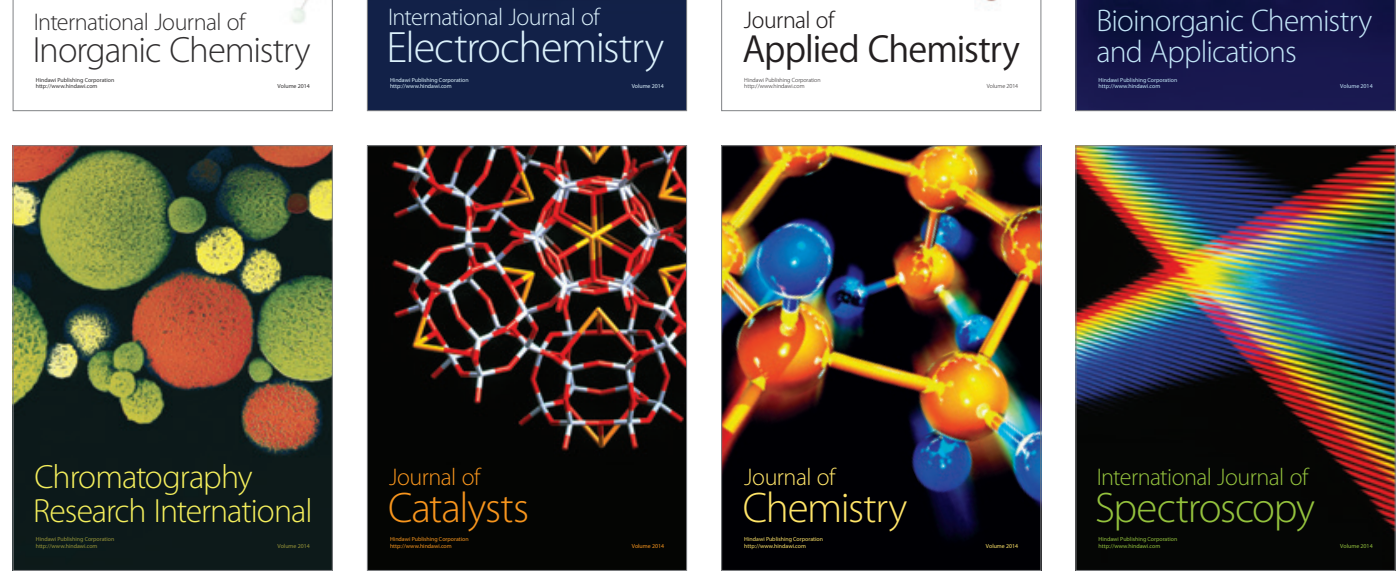